\title{
RELAÇÃO DO PERFIL ACADÊMICO DOS PROFESSORES DA UNIDADE ESCOLAR GOVERNADOR PEDRO FREITAS COM AS ESTRATÉGIAS DE AVALIAÇÃO DA APRENDIZAGEM DE ALUNOS DE ENSINO MÉDIO ${ }^{1}$
}

Karllos Augusto Sampaio Junior ${ }^{2}$

\section{RESUMO}

Este trabalho balizou-se pelo problema: Qual a relação do perfil acadêmico dos professores da Unidade Escolar Governador Pedro Freitas com as estratégias de avaliação aprendizagem de alunos de ensino médio? Objetivou-se no geral a determinar a relação do perfil acadêmico dos professores da Unidade Escolar Governador Pedro Freitas com as estratégias de avaliação da aprendizagem de alunos do ensino médio, especificamente objetivou-se a descrever o perfil acadêmico dos professores, a averiguar o conhecimento dos professores sobre avaliação da aprendizagem, a identificar os tipos, métodos e dinâmicas de avaliação da aprendizagem utilizadas pelo professor e a verificar a percepção dos professores quanto à atividade de avaliação da aprendizagem que utilizam com seus alunos. Com modelo não experimental, tipo descritivo na modalidade estudo de caso e enfoque qualitativo, foi utilizada uma população de 44 sujeitos de análise, com autorização formal do (a) diretor (a) administrativo (a) pedagógico (a) como instrumento de coleta de dados foi utilizado um questionário semiestruturado aos professores. A maioria dos professores iniciou sua formação em curso superior, acessou ou estão acessando alguma pós-graduação predominantemente em especialização. Averiguou-se que um pequeno número de professores já acessou ou acessa formação específica com conhecimento pleno sobre avaliação da aprendizagem. Identificou-se que os professores de forma descontínua e divergente escolhem as dinâmicas de avaliação da aprendizagem. Verificou-se que a maioria dos professores concebe como ideia de avaliação o ato de examinar com objetivos formativos e consideram o contexto social, econômico e histórico dos alunos no ato de avaliarem suas aprendizagens.

Palavras Chave: Aprendizagem. Avaliação. Formação. Prática Docente.

\footnotetext{
${ }^{1}$ Trabalho apresentado no Congresso Brasileiro Ciência e Sociedade (CBCS 2019), promovido pelo Centro Universitário Santo Agostinho, de 03 a 05 de outubro de 2019, em Teresina-PI.

${ }^{2}$ Mestre em Ciências da Educação, Universidad Autónoma de Asunción - PY, UEGPF, email: real123456789realidade@gmail.com
} 


\title{
RELATION OF THE ACADEMIC PROFILE OF TEACHERS OF THE GOVERNOR PEDRO FREITAS SCHOOL UNIT WITH LEARNING SCHOOL LEARNING STRATEGIES
}

Karllos Augusto Sampaio Junior

\begin{abstract}
This work was marked by the problem: What is the relationship of the academic profile of the teachers of the Governor Pedro Freitas School Unit with the strategies of learning evaluation of high school students? The general objective was to determine the relationship between the academic profile of the teachers of the Governor Pedro Freitas School Unit and the strategies for learning evaluation of high school students, specifically to describe the academic profile of teachers, to ascertain the knowledge of the teachers. teachers on learning evaluation, to identify the types, methods and dynamics of learning evaluation used by the teacher and to verify teachers' perceptions of the learning evaluation activity they use with their students. With a non-experimental model, descriptive type in the case study and qualitative approach, a population of 44 analysis subjects was used, with formal authorization from the pedagogical administrative director as an instrument for collecting data. Data was used a semi-structured questionnaire to teachers. Most teachers have started their college education, have accessed or are accessing some predominantly postgraduate specialization. It was found that a small number of teachers have already accessed or access specific training with full knowledge about learning evaluation. It was identified that teachers discontinuous and divergent choose the dynamics of learning evaluation. It was found that most teachers conceive as an idea of evaluation the act of examining with formative goals and consider the social, economic and historical context of students in the act of evaluating their learning.
\end{abstract}

Keywords: Learning. Evaluation. Formation. Teaching Practice.

\section{INTRODUÇÃO}

Avaliar é parte da ação pedagógica que por sua vez faz parte da ação humana, requerendo leitura, reflexão, debate, revisão de conceitos e prática. A ação de avaliar sempre denota julgamento, definição de algo, de mentes com comportamentos e estados psicológicos diferentes, onde estão inclusos os docentes.

Com o problema: Qual a relação do perfil acadêmico dos professores da Unidade Escolar Governador Pedro Freitas com as estratégias de avaliação aprendizagem de alunos de ensino médio? O objetivo geral foi de determinar a relação do perfil acadêmico dos professores da Unidade Escolar Governador Pedro Freitas com as estratégias de avaliação da aprendizagem de alunos de ensino médio e como objetivos 


\section{CONGEESSOC CIENCIAESOCIEDADE

ANAIS CBCS 2019 | 3 a 5 de outubro de 2019 | Centro Universitário Santo Agostinho - Teresina - P|

específicos a descrever o perfil acadêmico dos professores, a averiguar o conhecimento dos professores sobre avaliação da aprendizagem, a identificar os tipos, métodos e dinâmicas de avaliação da aprendizagem utilizadas pelo professor e a verificar a percepção dos professores quanto à atividade de avaliação da aprendizagem que utilizam com seus alunos

Referenciou-se por teóricos tais como: Alves (2013), Antonio (2002), Ghiraldelli (1991), Imbernón (2011), LDBs 9394/96 Brasil (2010), Perrenoud (2002), Sacristán y Goméz (2007), Sant'Anna (2014)

Com isto esta pesquisa possibilitará uma reflexão sobre a avaliação da aprendizagem ligada diretamente a qualidade de formação e da prática do docente, analisando seus detalhes, suas bases filosóficas, científicas e pedagógicas transformando assim a escola e suas atividades em momentos de real significado para a vida dos sujeitos que a compõem.

\section{REFERENCIAL TEÓRICO}

A formação docente permeia-se com o objetivo de conquista de um espaço profissional amplo, de não exclusão social, de colaboração com e para a comunidade e garantia democrática de estabelecer sua valorização como profissão e como profissional. (IMBERNÓN, 2011).

Perrenoud (2002) instiga a reflexão docente na necessidade de inter-relacionar e contextualizar o indivíduo ao seu nicho de vida, representado por seu ambiente familiar, cultural, seu histórico de vida.

Paralela a esta formação urge-se entender em qual foco a prática avaliativa, por suas bases teóricas e metodológicas são abordadas, considerando sua vasta complexidade e divergências de conceitos.

Em 1991 Ghiraldelli Jr. destacou que no Brasil as tendências pedagógicas dos anos iniciais traziam em si uma característica da metodologia jesuítica com forte teor tradicional, forte apelo à memorização. 


\section{CONGEESSOC CIENCIAESOCIEDADE

ANAIS CBCS 2019 | 3 a 5 de outubro de 2019 | Centro Universitário Santo Agostinho - Teresina - P|

A partir de 1985, contexto brasileiro sofre transformações institucionais, influenciando o comportamento social e político; o ensino bem como suas práticas, por novas reflexões assumem um novo caráter formador ensejando também em uma nova perspectiva avaliativa. A avaliação da aprendizagem assume um caráter mais contextualizado, interdisciplinar, resultando em um conhecimento vasto. (ANTÔNIO, 2002).

Alves (2013) e Sacristán (2007) destacam uma avaliação da aprendizagem com valorização dos aspectos construtivistas priorizando as situações do ambiente e suas relações com as situações problemas, destacando ainda o valor cooperativo dos alunos e classificam por ela a condição de se fazer política educativa e estabelecer amplamente a educação.

Uma gama de estratégias avaliativas ganha espaço, preservando as particularidades, individualidades específicas com o fino objetivo de bem avaliar, tais como: atividades de classe, observações, relatórios, pré-testes e promoção de autoavaliação com o aluno. (SANT' ANNA, 2014).

\section{METODOLOGIA}

Este trabalho com autorização do (a) diretor (a) administrativo (a) e pedagógica (o) da referida instituição, adotou concepção ou desenho não experimental, de concepção transversal descritivo onde apenas observou-se o momento em que o fato ocorreu sem, contudo intervir nos fatos observados. (SAMPIERI. et al., 2013).

Optou-se pelo alcance descritivo, buscou especificar as propriedades, as características ou qualquer outro fenômeno que se submeta a uma análise, adotou-se o enfoque qualitativo, pois a intenção foi compreender a perspectiva do sujeito ou unidade de análise com paradigma de estudo de caso. (ARANDA, 2018).

Utilizou-se o número da população dos sujeitos em sua totalidade, 44 sujeitos, formando assim a unidade de análise, como o número de sujeitos foi menor que 100, não foram utilizadas amostras, considerando assim toda a população, (SAMPIERI. et al. 2013). 


\section{CONGEESSOC CIENCIAESOCIEDADE \\ Inovação, Diversiliade e Sustentahilitiade}

ANAIS CBCS 2019 | 3 a 5 de outubro de 2019 | Centro Universitário Santo Agostinho - Teresina - P

Os instrumentos de coleta de dados basearam-se no questionário semiestruturado, com perguntas onde o questionado poderia optar por alternativa de respostas fechadas ou propostas pelo questionados, validados por juízo de especialistas através de recomendações específicas. (ARANDA, 2018).

\section{RESULTADOS E DISCUSSÃO}

\section{Variável Formação Docente}

\section{Questionário Semiestruturado Aplicado aos Professores}

\section{Perfil acadêmico e de formação inicial e continuada dos professores}

Fig.1 - Formação inicial profissional dos professores

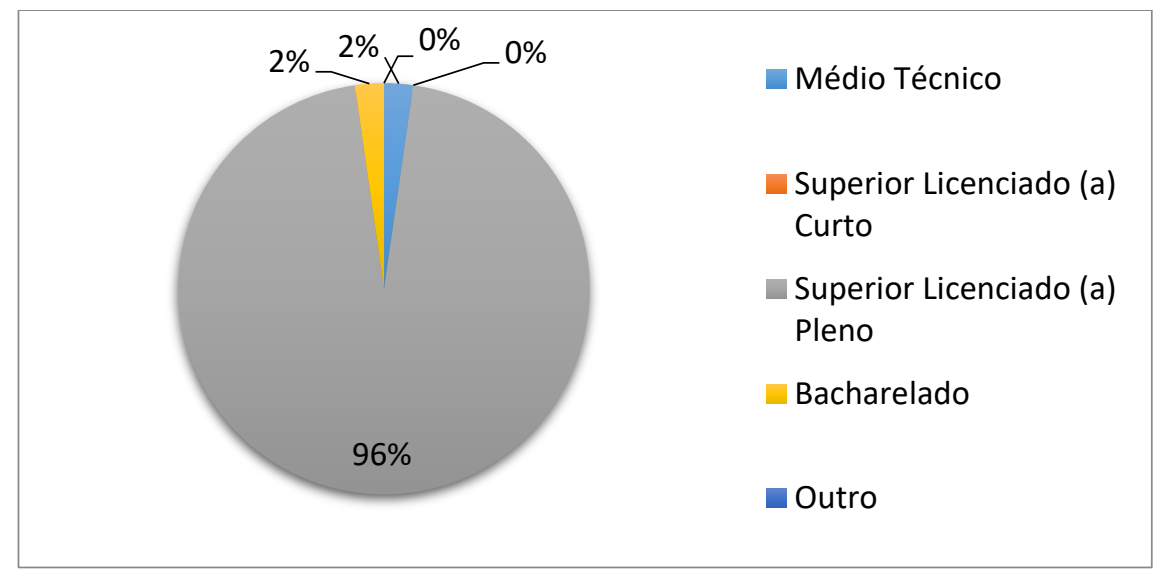

FONTE: Dados da Pesquisa

Dos professores participantes, 96\% respondeu ter iniciadas suas formações no ensino superior em nível de graduação em licenciatura plena.

Fig. 2 - Tipo de pós-graduação que os professores cursaram ou cursam

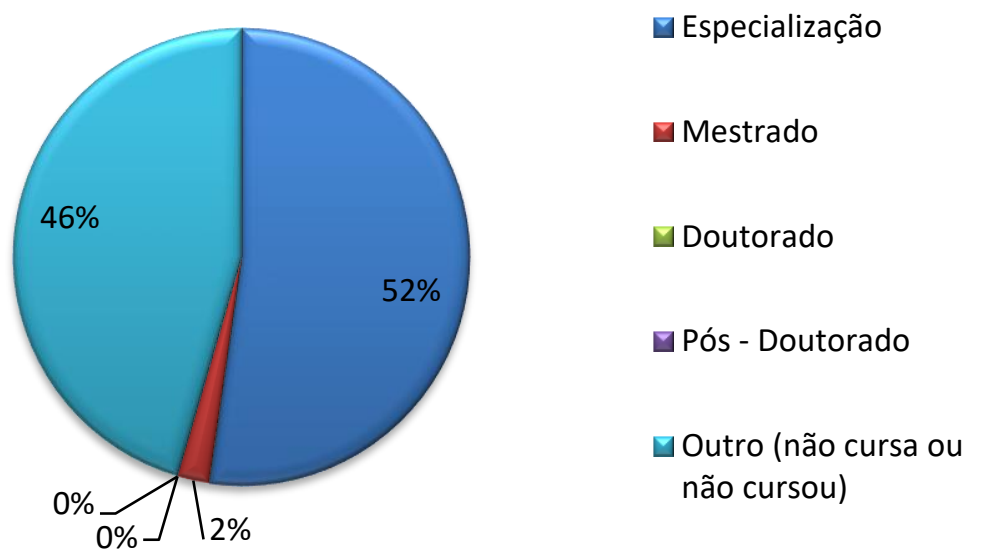




\section{CONGEESSOC CIENCIAESOCIEDADE \\ Inovação, Diversiliade e Sustentahilitiade}

ANAIS CBCS 2019 | 3 a 5 de outubro de 2019 | Centro Universitário Santo Agostinho - Teresina - P

FONTE: Dados da Pesquisa

Dos professores participantes, 52\% responderam ter continuado suas formações profissionais ao menos em nível de pós-graduação no tipo especialização, 46\% não cursou ou não está cursando.

\section{- Averiguação do conhecimento dos professores sobre avaliação da aprendizagem}

Fig.3 - Foco em avaliação da aprendizagem dos professores em suas formações acadêmicas

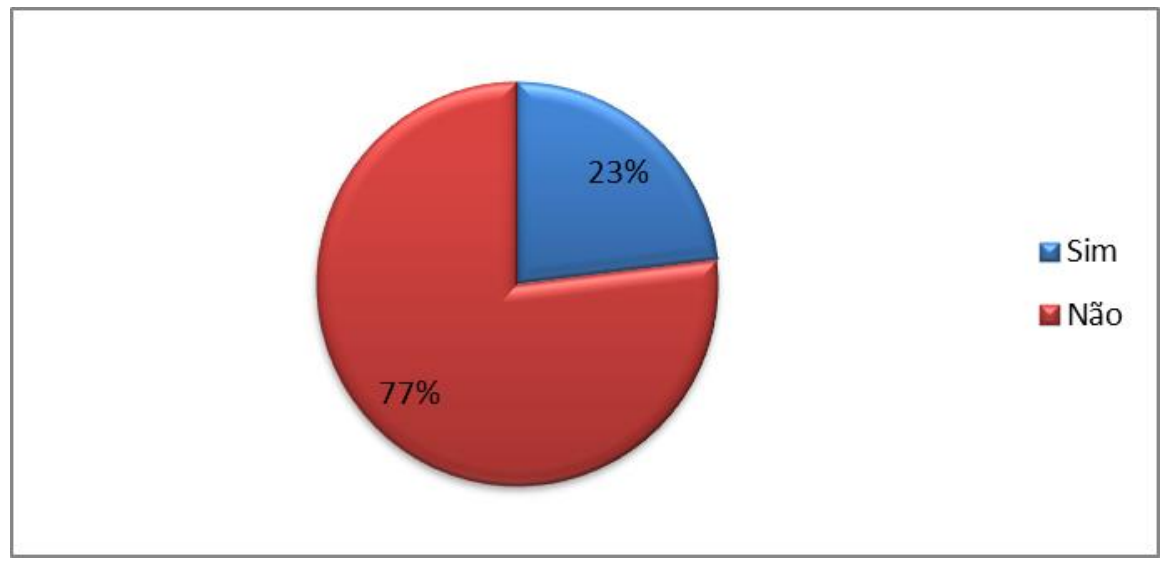

FONTE: Dados da Pesquisa

Dos professores participantes, $77 \%$ respondeu que não houve foco sobre a temática avaliação da aprendizagem, enquanto $23 \%$ respondeu que houve.

Fig. 4 - Participação dos professores em formação específica sobre avaliação

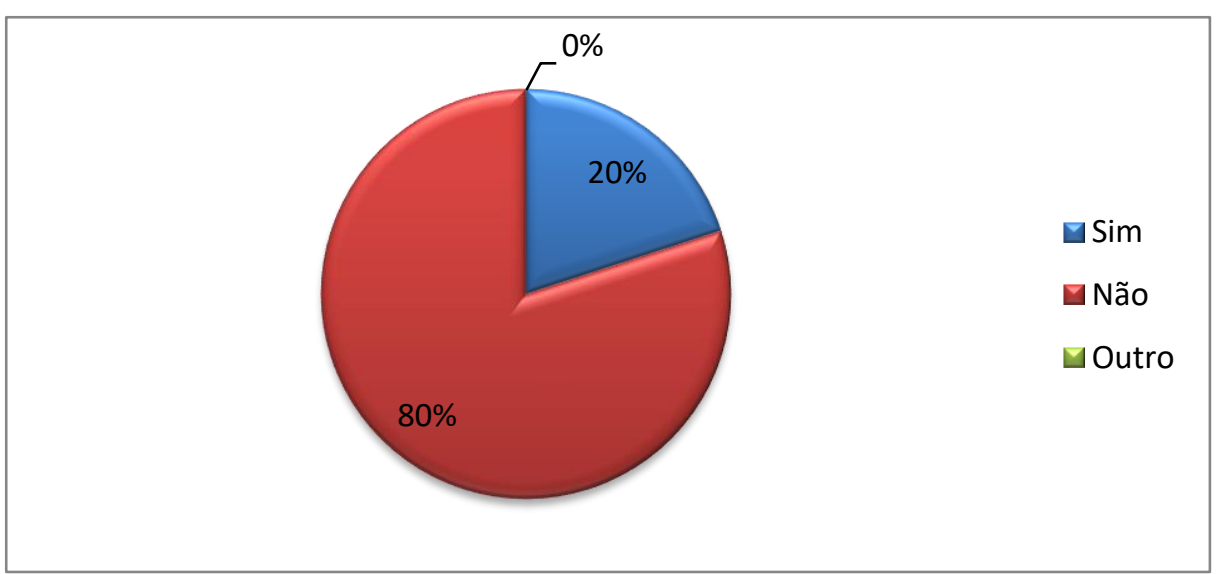

FONTE: Dados da Pesquisa

Dos professores participantes, 80\% respondeu "não", 20\% respondeu "sim".

Fig. 5 - Identificação do conhecimento técnico dos professores sobre avaliação da aprendizagem 


\section{CONGEESSOC CIENCIAESOCIEDADE \\ Inovaçãa, Diversidaade e Sustentahililitaile}

ANAIS CBCS 2019 | 3 a 5 de outubro de 2019 | Centro Universitário Santo Agostinho - Teresina - P

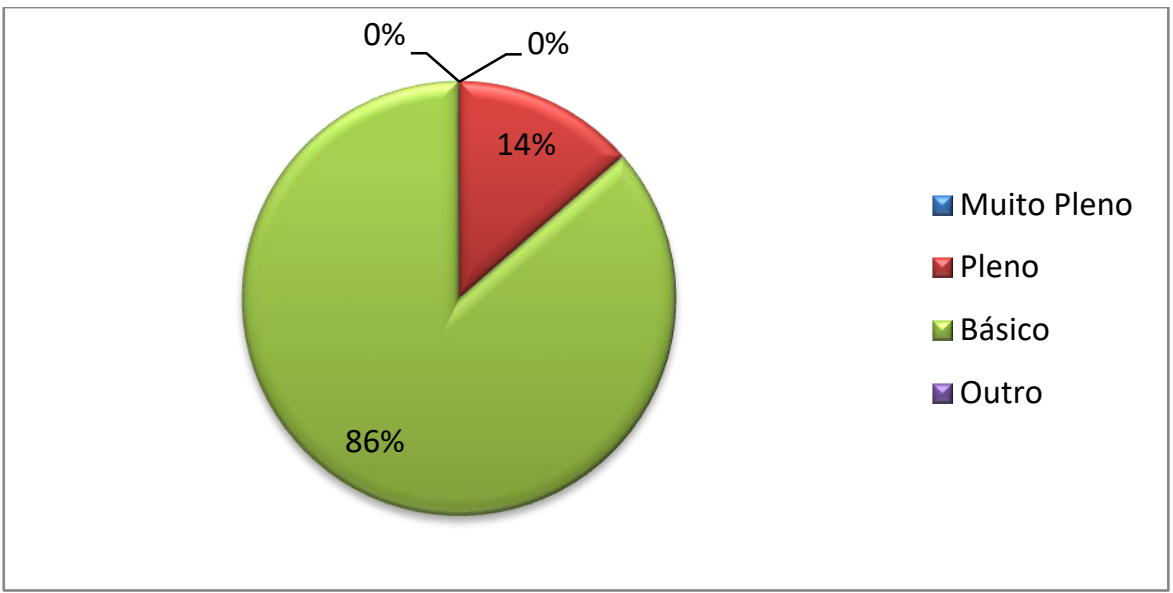

FONTE: Dados da Pesquisa

Dos professores participantes, $86 \%$ respondeu ter conhecimento técnico básico, ao passo que $14 \%$ conhecimento técnico pleno sobre avaliação da aprendizagem.

\section{- Variável Avaliação da Aprendizagem}

- Identificação dos tipos, métodos e dinâmicas de avaliação da aprendizagem utilizadas pelos professores.

Fig. 6 - Tipo de avaliação da aprendizagem que os professores utilizam

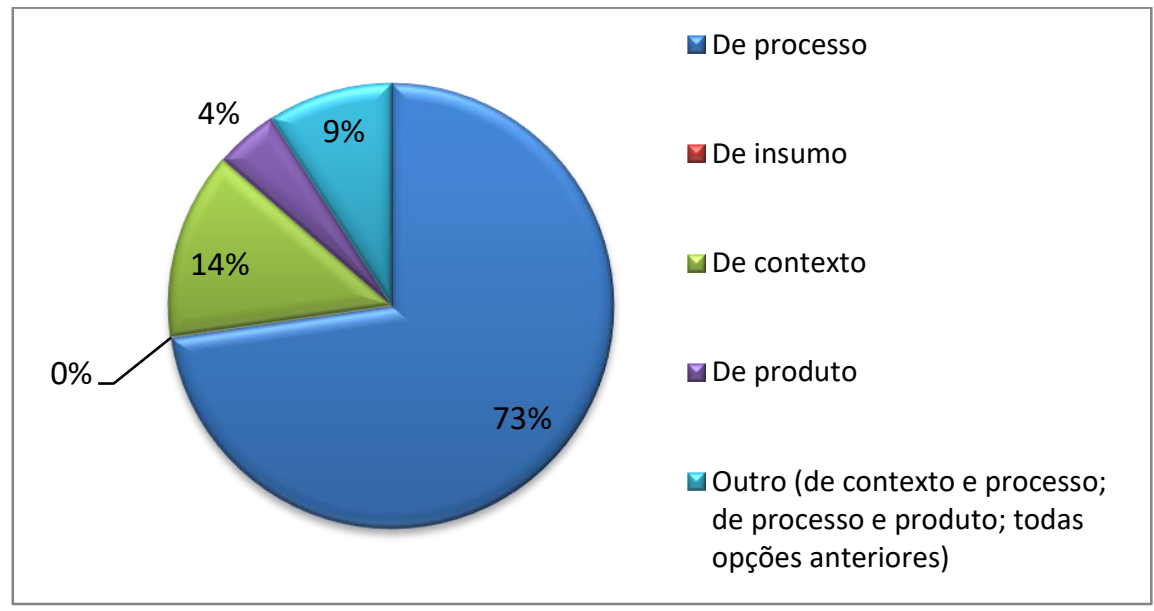

FONTE: Dados da Pesquisa

Dos professores participantes, $73 \%$ respondeu que utilizam as avaliações do tipo processo.

Fig. 7- Método utilizado pelos professores na aplicação das avaliações da aprendizagem 


\section{CONGEESSOC CIENCIAESOCIEDADE \\ Inovaçãa, Diversidaade e Sustentahililitaile}

ANAIS CBCS 2019 | 3 a 5 de outubro de 2019 | Centro Universitário Santo Agostinho - Teresina - P

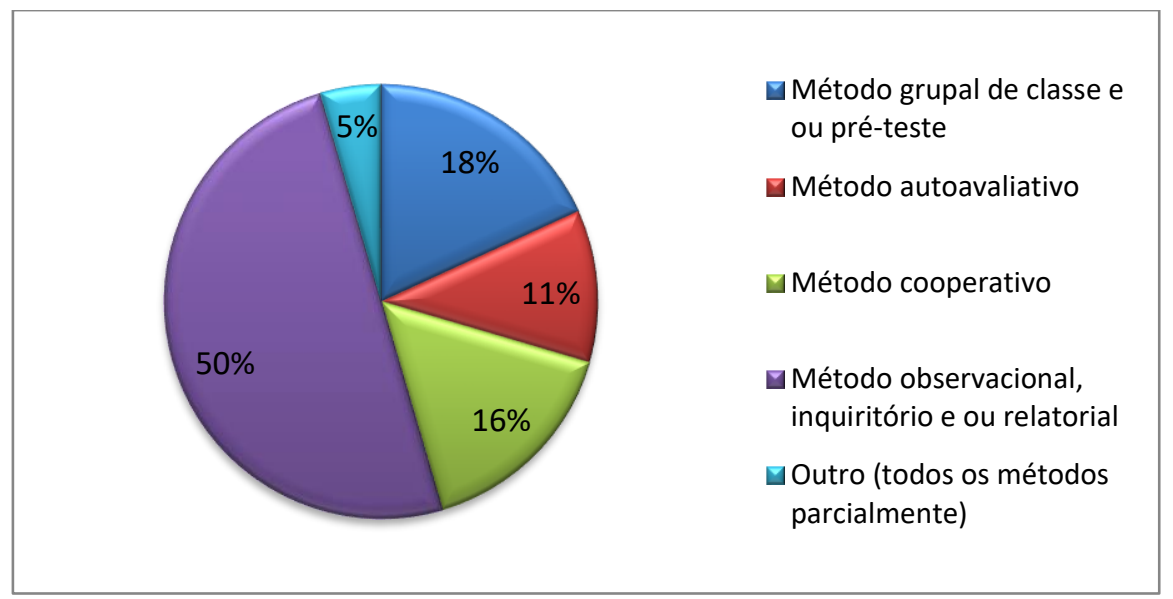

FONTE: Dados da Pesquisa

Dos professores participantes, 50\% respondeu utilizar o método observacional, inquiritório e ou relatorial; $18 \%$ o método grupal de classe e ou pré-teste, $16 \%$ o método cooperativo, $11 \%$ o método autoavaliativo e $5 \%$ todos os métodos.

Fig. 8 - Dinâmica utilizada pelos professores na aplicação da avaliação da aprendizagem

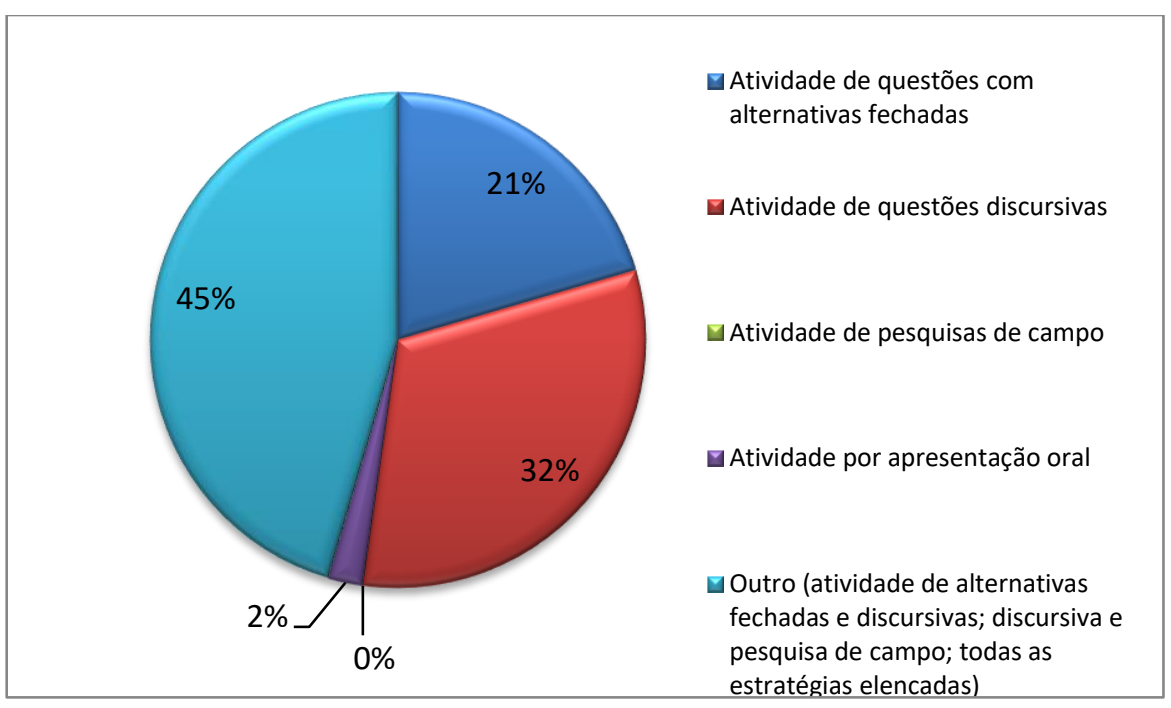

FONTE: Dados da Pesquisa

Dos professores participantes, $45 \%$ responderam: "atividades de alternativas fechadas e discursivas"; "discursivas e pesquisa de campo", todas as estratégias elencadas"; $32 \%$ atividade de questões discursivas, $21 \%$ atividade de questões com alternativas fechadas, $2 \%$ atividade por apresentação oral.

\section{- Variável Estratégia Docente}




\section{CONGEESSOC CIENCIAESOCIEDADE \\ Inovaçãa, Diversidaade e Sustentahililitaile}

ANAIS CBCS 2019 | 3 a 5 de outubro de 2019 | Centro Universitário Santo Agostinho - Teresina - P

- Verificação da percepção dos professores quanto à avaliação da aprendizagem que utilizam com seus alunos

Fig. 9 - Verbo que melhor traz uma percepção dos professores sobre avaliação da aprendizagem

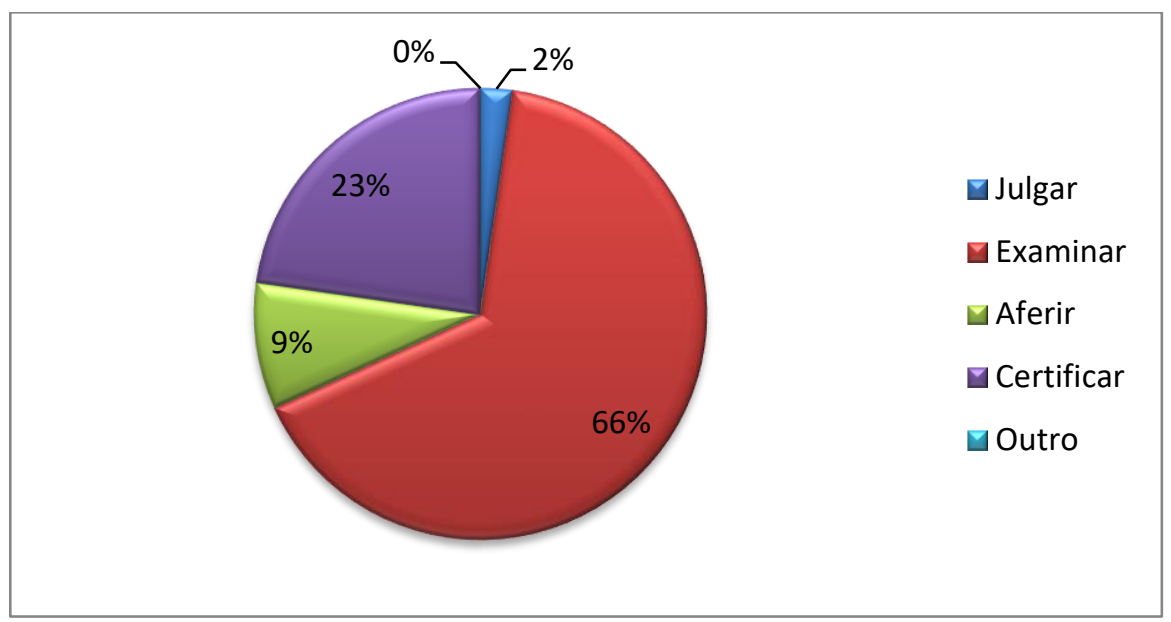

FONTE: Dados da Pesquisa

Dos professores participantes, $66 \%$ percebe através do verbo examinar, $23 \%$ através do verbo certificar, $9 \%$ através do verbo aferir, $2 \%$ do verbo julgar.

Fig. 10 - Percepção dos professores após a realização da avaliação da aprendizagem

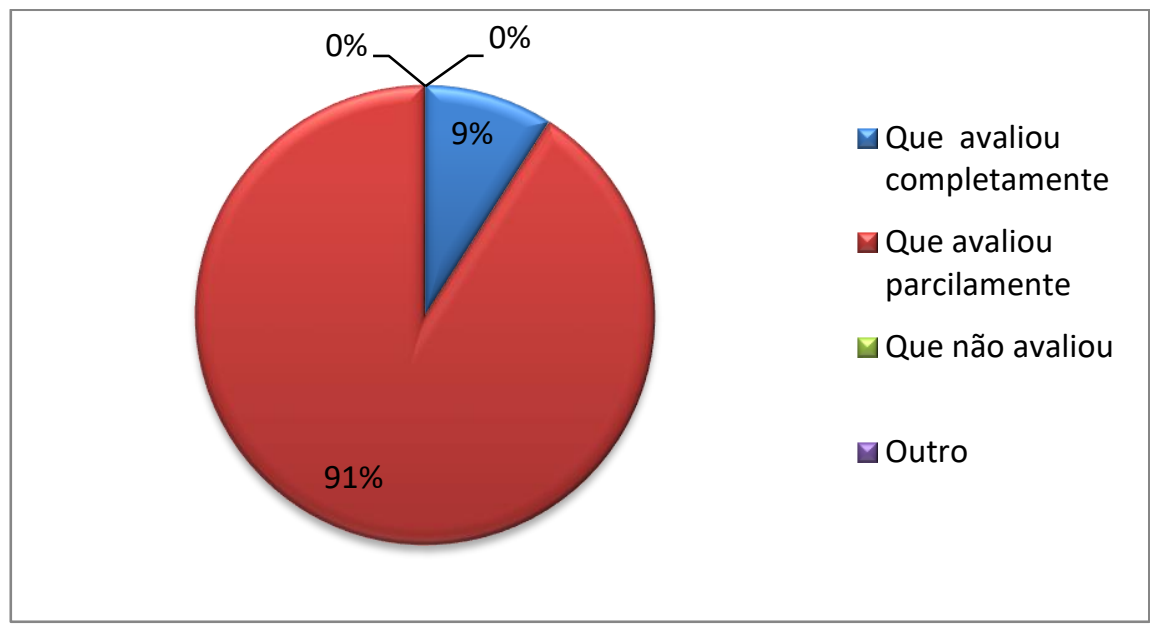

FONTE: Dados da Pesquisa

Dos professores participantes, $91 \%$ responderam ter avaliado a aprendizagem parcialmente, $9 \%$ respondeu ter avaliado completamente.

\section{CONSIDERAÇÕES FINAIS}

Variável Formação Docente 


\section{CONGESSOCOENENAESOCIEDADE Inovaçãa, Diversidaade e Sustentahililitaile}

ANAIS CBCS 2019 | 3 a 5 de outubro de 2019 | Centro Universitário Santo Agostinho - Teresina - P

\section{Descrever o perfil acadêmico e de formação inicial e continuada dos professores}

Descreve-se que a maioria dos docentes formaram-se inicialmente em licenciatura plena e nos quais $54 \%$ destes docentes acessaram ou estão acessando alguma pósgraduação predominantemente em especialização.

\section{Averiguar o conhecimento dos professores sobre avaliação da aprendizagem}

Averiguou-se que na maioria de suas formações não houve um foco na abordagem em avaliação da aprendizagem, não participam de qualquer formação específica em avaliação e consideram-se com apenas conhecimento básico sobre o tema.

\section{Variável Avaliação da Aprendizagem}

Identificar os tipos, métodos e dinâmicas de avaliação da aprendizagem utilizadas pelos professores

Identificou-se que fazem opção pela avaliação da aprendizagem do tipo processo, porém dividem-se na utilização de métodos, com predomínio do observacional inquiritório e ou relatorial, bem como na escolha das dinâmicas com "atividades de alternativas fechadas e discursivas"; "discursivas e pesquisa de campo", todas as estratégias elencadas".

\section{Variável Estratégia Docente}

Verificar a percepção dos professores quanto à avaliação da aprendizagem que utilizam com seus alunos

Verificou-se predominantemente os docentes associam ao verbo examinar ao processo de avaliação da aprendizagem e entendem que avaliam parcialmente seus alunos.

Portanto a relação do perfil acadêmico dos professores da referida unidade de ensino com as estratégias de avaliação da aprendizagem que utilizam com seus alunos de ensino médio seguem diretamente relacionada às suas formações iniciais e continuadas, bem como a praticamente ausência de formação institucional sobre o tema, o que corrobora na heterogeneidade quanto a escolha de tipos, métodos e dinâmicas escolhidas; embora a concepção de exame predomine quanto ao tema 


\section{CONGEESSOC CIENCIAESOCIEDADE \\ Inovaçãa, Diversiliadle e Sustentalililitaile}

ANAIS CBCS 2019 | 3 a 5 de outubro de 2019 | Centro Universitário Santo Agostinho - Teresina - P

avaliativo o grau de parcialidade no que avaliam seja latente proporcionando uma fragmentação com o conteúdo ensinado.

\section{REFERÊNCIAS}

ALVES, J. F. Avaliação Educacional: Da Teoria à Prática. Rio de Janeiro. RJ: LTC. 2013.

ANTÔNIO, S. Educação e Transdisciplinaridade: a necessidade de uma nova "escola poética. Rio de Janeiro: RJ. Lucerna. 2002.

ARANDA, T. J. C. Metodología De La Investigación Científica: Manual Para Elaboración de Tesis y Trabajos de Investigación. Asunción: PY. Marben Editora. 2018.

BRASIL, L. D. Lei de Diretrizes e Bases da Educação Nacional: lei 9394, de 20 de dezembro de 1996, que estabelece as diretrizes da educação nacional. Brasília: DF. Edições Câmara. 2010

GHIRALDELLI, Jr., P. História da Educação. São Paulo: SP. Cortez. 1991

IMBERNÓN, F. Formação Docente e Profissional: Formar-se Para A Mudança E A Incertezas. São Paulo. SP: Cortez Editora. 2011.

PERRENOUD, P. A Prática Reflexiva no Ofício de Professor: Profissionalização e Razão Pedagógica. Porto Alegra: RS. Artmed. 2002.

SAMPIERI, R. H.; COLADO, C. H.; LUCIO, P. H. B. Metodologia de Pesquisa. Porto Alegre. RS: Penso. 2013

SANT'ANNA, I. M. Por Que Avaliar? Como Avaliar? Critérios e Instrumentos. Petrópolis. RJ: Vozes. 2014.

SACRISTÁN, J. G.; GÓMEZ, A. I. P. Compreender e transformar o ensino. São Paulo. SP: Artmed. 2007. 